\title{
Oferta de Trabalho Comparada nas Regiões Metropolitanas de São Paulo, Curitiba e Porto Alegre
}

\section{Comparative Labor Supply in Metropolitan Areas of São Paulo, Curitiba and Porto Alegre}

Silvana Maria do Amaral Moraes ${ }^{1}$

Gustavo Inácio de Moraes²

Resumo: O objetivo deste artigo é estabelecer como as características pessoais influenciam na oferta de trabalho dos trabalhadores das Regiões Metropolitanas de São Paulo, Curitiba e Porto Alegre. Foi realizada uma estimação em pooling para os anos de 2001 a 2005, utilizando o procedimento de Heckman, com base nos microdados da PNAD. É demonstrado, empiricamente, um resultado de acordo com a teoria, exceto para a variável idade. Foi confirmado que o número de filhos que residem junto com a mulher é uma variável importante para determinar a participação da mesma no mercado de trabalho, mas não para definir a quantidade de horas trabalhadas.

Palavras-chave: Oferta de trabalho. Emprego urbano. Procedimento de Heckman.

Abstract: This paper examines and measures how personal characteristics reflect in supply labor in Sao Paulo, Porto Alegre and Curitiba metropolitan region. For this purpose we utilized data from PNAD (IBGE) in 2001-2005 period; we constructed pooling estimation applying Heckman's procedure. Empirical results confirms theoretical predictions for labor market, except to age variable, and confirms importance of number of children for woman entry in labor market despite this variable is not significant for choose hours worked.

Keywords: Labor supply. Urban employment. Heckman`s Procedure.

JEL Classification: C01; J22.

\section{Introdução}

A modificação dos padrões de desenvolvimento econômico e a inconstância da conjuntura econômica ocasionam alterações significati-

1 Doutoranda em Economia Aplicada - ESALQ/ USP. Mestra em Desenvolvimento Econômico (UFPR). E-mail: smmoraes@esalq.usp.br

2 Doutorando em Economia Aplicada - ESALQ/USP. Mestre em Desenvolvimento Econômico (UFPR).E-mail: moraes@esalq.usp.br 
vas no mercado de trabalho brasileiro. As regiões metropolitanas de São Paulo, Curitiba e Porto Alegre permanecem, neste início do século XXI, como pólos concentradores de mão-de-obra e apresentam dinamismo nos setores industriais e de serviços. Essas metrópoles se mantêm em destaque, como regiões cujas áreas de influência se estendem por todo o território brasileiro e até mesmo por outros países, como no caso de São Paulo.

As tendências verificadas nessas três regiões sinalizam expressiva parcela daquilo que acontece no restante do País. Justificando, basta notar que no estado de São Paulo, dos cinco municípios com maior PIB, quatro deles estão na Região Metropolitana; no estado do Paraná, os três primeiros localizam-se na Região Metropolitana de Curitiba e no Rio Grande do Sul, quatro dos cinco maiores produtos municipais estão na Região Metropolitana de Porto Alegre. Como consequência, os municípios que compõem as regiões metropolitanas de São Paulo, Curitiba e Porto Alegre são responsáveis por, respectivamente, 32,9\%, 35,1\% e 40,3\% do produto estadual (IBGE, 2006).

Os reflexos da conjuntura econômica são sentidos de forma distinta, ao mesmo tempo em que os estágios de desenvolvimento das três regiões metropolitanas encontram-se diferenciados. Portanto, seria de esperar distinção entre a característica da oferta de trabalho entre uma região metropolitana e outra.

O objetivo deste artigo é examinar os fatores que influenciam a oferta de trabalho e a intensidade com que se manifestam, distinguindo os trabalhadores por três regiões metropolitanas (Curitiba, Porto Alegre e São Paulo) e por gênero, nos anos compreendidos entre 2001 e 2005, a partir dos dados da PNAD.

Para atingir o objetivo proposto, o artigo divide-se em quatro seções, além desta introdução. Na segunda seção serão explicitados os aspectos teóricos que nortearão este artigo e, na terceira, será apresentada a metodologia utilizada, destacando-se o procedimento de Heckman. Na quarta parte do artigo, os resultados da estimação serão apresentados e discutidos e, finalmente, na última seção, serão expostos os comentários finais.

\section{Oferta de Trabalho - Aspectos Teóricos}

A Teoria da Oferta de Trabalho analisa a decisão do indivíduo em participar do mercado de trabalho, bem como escolher a melhor alocação de horas trabalhadas e horas de lazer, levando-se em conta o salário ofertado. Nesse sentido, a estrutura de preferências do trabalhador é decisiva na escolha entre horas de lazer e trabalho. Dessa maneira, podem ser identificados dois efeitos: o efeito-renda e o efeito-substituição. A decisão de participação no mercado de trabalho dependerá das 
preferências individuais e/ou das preferências familiares, considerando todos os membros da família.

Desse modo, o objetivo do consumidor é maximizar a função utilidade $u(q, l)$, no qual $q$ é o consumo agregado do indivíduo e $l$ as horas de lazer do indivíduo. É necessário, contudo, obedecer às seguintes restrições, conforme aponta Barbosa (1985):

Maximizar $u(q, l)$, sujeito a:

$$
\begin{aligned}
& p q \leq w h+R \\
& h+1 \leq t \\
& q \geq 0, h \geq 0 \text { e } 1 \geq 0
\end{aligned}
$$

No qual,

$p=$ índice de preços de consumo;

$q=$ índice de quantidade de produtos consumidos;

$w=$ salário-hora recebido pelo indivíduo;

$h=$ número de horas trabalhadas pelo indivíduo;

$R=$ rendimento de outras fontes no período de referência;

$l$ = número de horas dedicadas ao lazer no período de referência;

$t=$ número de horas totais no período de referência.

O efeito-renda atua, predominantemente, no sentido de reduzir as horas ofertadas de trabalho, uma vez que para um dado nível de renda são necessárias menos horas de trabalho. Concomitantemente, atua o efeito substituição que, sob alta no salário, atua principalmente no sentido de serem ofertadas mais horas de trabalho, uma vez que o custo de oportunidade das horas de lazer elevou-se.

O resultado líquido é, a princípio, incerto se considerado a somatória dos dois efeitos. Entretanto, as estimações anteriores disponíveis na literatura mostram que para níveis menores de salário, o efeito-substituição é dominante em relação ao efeito-renda, tornando a curva de oferta de trabalho positivamente inclinada no plano das horas trabalhadas e salário. Em determinado nível de salário, contudo, o efeito-substituição deixa de ser dominante, passando a ser dominado pelo efeito-renda, o que torna a curva de oferta de trabalho negativamente inclinada (backward bending). Não se descarta, ainda, a possibilidade de a curva de oferta de trabalho possuir faixas de variações no qual ora predomina o efeito-substituição, ora predomina o efeito-renda, dependendo das preferências apresentadas pelo indivíduo.

Um caso importante que merece destaque é a "solução de canto", que representa a indisponibilidade de horas de trabalho e a opção apenas por horas de lazer. Em outros termos, indica que o salário que o indivíduo atribui às suas horas (preço-sombra) encontra-se acima daquele praticado no mercado, sendo que tal ocorrência corresponderia ao indivíduo parti- 
cipar ou não do mercado de trabalho. Outra "solução de canto" possível é a decisão em alocar todas as horas disponíveis para o trabalho, recusando horas de lazer. No entanto, tal equilíbrio é possível apenas no aspecto teórico, já que indivíduo algum será capaz de desistir das horas de sono.

Um tratamento proposto por Heckman $(1974 ;$ 1979) considera conjuntamente os indivíduos que se encontram na "solução de canto" e os indivíduos cujo preço-sombra é igual ou menor ao praticado no mercado, ou seja, os indivíduos que ofertam horas de trabalho. Nessa abordagem, o indivíduo deseja maximizar $u(q, l)$, mas oferta um número fixo de horas, $h_{\mathrm{f}}$. Sendo assim, a condição de maximização não permite mais uma "solução de canto" e, portanto, seria possível encontrar o salário de reserva daqueles indivíduos que não participam do mercado de trabalho, através da solução da equação com restrições, relacionando-o com o salário observado no mercado. Pela notação de Barbosa (1985), formalmente:

Maximizar $u(q, l)$, sujeito a:

$$
\begin{aligned}
& p q \leq w h+R \\
& h_{\mathrm{f}}+1 \leq t \\
& q \geq 0, h_{\mathrm{f}} \geq 0 \text { e } l \geq 0
\end{aligned}
$$

Onde,

$p=$ índice de preços de consumo;

$q$ = índice de quantidade de produtos consumidos;

$w=$ salário-hora recebido pelo indivíduo;

$h_{\mathrm{f}}=$ número de horas trabalhadas pelo indivíduo;

$R=$ rendimento de outras fontes no período de referência;

$l=$ número de horas dedicadas ao lazer no período de referência;

$t=$ número de horas totais no período de referência.

Como lembram ainda Killingsworth e Heckman (1986), é possível encontrar três tipos de erros relacionados à estimação das horas de trabalho. Eles seriam associados à função utilidade, à restrição orçamentária e à localização do ponto de ótimo. Os erros associados à otimização, referem-se à diferença entre o ótimo de horas ofertadas e as horas observadas de trabalho. Erros que dizem respeito à função utilidade e à restrição orçamentária, referem-se a diferenças não observáveis entre os indivíduos para as preferências e orçamentos individuais, respectivamente.

Deaton e Muellbauer (1991, p. 90-92) formalizam o mesmo modelo de oferta de horas de trabalho para demonstrar a possibilidade dos efeitos substituição e renda também não possuírem, a priori, predominância garantida. Entretanto, esses demonstram a viabilidade de obter tais informações por meio das funções custo e utilidade indireta.

Zylberstajn e Balbinotto Neto (1999) lembram que, com base na teoria do job search, o indivíduo procura o maior salário possível, mas, em 
sua busca, considera em paralelo os custos da obtenção desta demanda de trabalho. De tal modo, os custos e os benefícios são considerados com base nas informações sujeitas a uma busca específica no mercado. Como conclusão, seu salário de reserva também seria dependente dos custos de obter a colocação no mercado. Os mesmos autores destacam que a participação no mercado de trabalho pode ainda ser explicada por alguns mecanismos alternativos que complementariam a decisão, a saber: (I) pela teoria da sinalização, no qual o indivíduo prepara-se para assumir posições específicas, evitando determinadas segmentações do mercado; (II) pelas histereses, fenômeno associado à tendência e às condições gerais do mercado de trabalho; (III) pelo salário eficiência, no qual se remunera o trabalhador acima do seu salário de mercado, gerando desemprego involuntário e, finalmente, (IV) pelos deslocamentos setoriais, característicos de momentos de mudanças nas demandas de habilidades, por alguma razão conjuntural ou estrutural associada ao comportamento dos setores econômicos. Os autores ainda lembram que os determinantes do salário de reserva podem ser encontrados no confronto entre custos de oportunidade e benefícios do emprego, considerados também intertemporalmente. O job search envolvido estaria centrado, sobretudo, na taxa de desconto intertemporal percebida pelo indivíduo e, portanto, teria maior relação com o desemprego. É exatamente a ocorrência de job search que motivou o artigo de Heckman (1979), onde são desenvolvidos procedimentos econométricos, apresentados na seção subsequente, para captar empiricamente os indivíduos que não participam do mercado de trabalho, de acordo com as restrições (1), (2), (3), anteriormente apresentadas. O indivíduo optaria em não participar do mercado de trabalho se o seu salário de reserva for superior ao salário de mercado, sendo possível interpretar que no salário de reserva já estariam implícitos os custos relativos ao job search.

Heckman e MaCurdy (1991) aperfeiçoam a equação de Frisch, que considera a oferta de trabalho intertemporal, sem a necessidade de se observar diretamente a utilidade marginal da moeda, um item de difícil verificação empírica. Para tanto, a utilidade marginal da moeda de um determinado período pode ser escrita como uma função da taxa de juros, do salário obtido e da oferta de trabalho (DONNI, 2006).

Deaton e Muellbauer (1991) lembram que os trabalhadores propensos a considerar seu salário de reserva para ingresso no mercado de trabalho são principalmente aqueles cujo trabalho é secundário na família ou, em outras palavras, aqueles que não chefiam famílias. Quando o rendimento do trabalhador principal cresce, o salário de reserva do trabalhador secundário dentro da família tende a aumentar e viceversa. Ao mesmo tempo, segundo os autores, seria possível observar 
diferença entre o padrão de oferta feminino e masculino, dado que a mulher tende a possuir obrigações que demandariam maior tempo no lar, como, por exemplo, atenção às crianças. Desse modo, a condição dentro da família, chefe ou secundário, e a presença de crianças podem determinar resultados importantes na oferta de trabalho.

No tocante à oferta de trabalho no Brasil, Ramos e Brito (2004), analisando o período entre 1991 e 2002, constatam algumas tendências do mercado de trabalho das áreas metropolitanas nos anos analisados, tendo como referência a PME (Pesquisa Mensal de Emprego). Percebese, por exemplo, que o nível de ocupação metropolitano cresce entre os anos 2000 e 2002, fato atribuído pelos mesmos às implicações sobre o mercado de trabalho decorrentes da alteração de política cambial. Nesses mesmos anos, a taxa de participação metropolitana decaiu, oscilando entre valores de $58 \%$ e $55 \%$, enquanto para o rendimento médio, constata-se uma queda consistente até 2002, iniciada em 1999. Dois pontos importantes emergem da análise: primeiro relativo ao destaque da crescente participação das mulheres, "tendo preenchido cerca de 2/3 dos postos de trabalho gerados, em termos líquidos, nesse ínterim" (BARROS; BRITTO, 2004, p. 16). O segundo destaque refere-se à diminuição do número de trabalhadores com escolaridade inferior a quatro anos de estudo pari passu ao aumento do número de trabalhadores "com alguma formação universitária”.

\section{Metodologia}

\subsection{Procedimentos}

O artigo examinará as condições da oferta de trabalho dos trabalhadores das Regiões Metropolitanas de São Paulo, Curitiba e Porto Alegre, utilizando-se de regressões de dados dispostos em pooling para os anos de 2001 a 2005. A regressão em pooling considera que os parâmetros associados às variáveis independentes são estáveis para os anos selecionados e implica, ademais, que não são observadas mudanças de políticas ou mudanças relativas na produtividade da mão-de-obra suficientes para alterar significativamente os parâmetros. Os dados são empilhados formando uma única amostra para os anos considerados e o modelo é analisado conjuntamente, sem distinguir o período da observação.

Considerando que a base de dados é composta pelas mulheres e homens que participam ou não do mercado de trabalho, ou seja, que ofertam ou não horas de trabalho, é necessário utilizar o enfoque de Heckman (1980). Caso não fosse considerado, as estimativas de mínimos quadrados ordinários, como se sabe, produziriam resultados viesados. 
No procedimento de Heckman opera-se, inicialmente, criando uma variável censurada para a participação no mercado de trabalho, aqui representada por P. Na sequência, é reproduzida a exposição de Kassouf (1994) dessa metodologia:

$$
\mathrm{P}_{\mathrm{i}}^{*}=\xi \mathrm{Z}_{\mathrm{i}}+\mathrm{u}_{\mathrm{i}}
$$

Onde,

$\mathrm{P}^{*}{ }_{\mathrm{i}}=$ representa a participação no mercado de trabalho;

$Z_{\mathrm{i}}=$ corresponde ao vetor de variáveis independentes que determina a participação do indivíduo no mercado;

$\mathrm{u}_{\mathrm{i}}=$ erro aleatório.

Como salientado, trata-se de uma variável binária onde $\mathrm{P}^{*}{ }_{\mathrm{i}}$ não é diretamente observável, mas:

$$
\begin{aligned}
& P_{i}=1, \operatorname{se} P_{i}^{*}>0 \\
& P_{i}=0, \operatorname{se} P_{i}^{*} \leq 0
\end{aligned}
$$

Uma vez que $\mathrm{H}$ representa o número de horas ofertadas no mercado de trabalho:

$$
\mathrm{H}=\beta^{\prime} \mathrm{R}_{\mathrm{i}}+\Psi_{\mathrm{i}}
$$

Onde,

$\mathrm{R}_{\mathrm{i}}=$ representa o vetor de variáveis que determina o número de horas trabalhadas,

$\Psi_{\mathrm{i}}=$ erro aleatório.

Desse modo, pode-se observar apenas o número de horas trabalhadas quando $\mathrm{P}_{\mathrm{i}}^{*}>0$. Assumindo que $\mathrm{u}_{\mathrm{i}} \mathrm{e} \Psi_{\mathrm{i}}$ são erros com média zero, desvios padrão $\sigma_{u}$ e $\sigma_{\Psi}$ e correlação $\rho$, então:

$$
\begin{aligned}
& \mathrm{E}\left(\mathrm{H}_{\mathrm{i}} \mid \mathrm{H}_{\mathrm{i}} \text { é observado }\right)=\mathrm{E}\left(\mathrm{H}_{\mathrm{i}} \mid \mathrm{P}_{\mathrm{i}}^{*}>0\right)=\mathrm{E}\left(\mathrm{H}_{\mathrm{i}} \mid \mathrm{u}_{\mathrm{i}}>-\xi^{\prime} \mathrm{Z}_{\mathrm{i}}\right) \\
& =\beta^{\prime} \mathrm{R}_{\mathrm{i}}+\mathrm{E}\left(\Psi_{\mathrm{i}} \mid \mathrm{u}_{\mathrm{i}}>-\xi^{\prime} \mathrm{Z}_{\mathrm{i}}\right)=\beta^{\prime} \mathrm{R}_{\mathrm{i}}+\rho \sigma_{\mathrm{\psi}} \lambda_{\mathrm{i}}\left(\alpha_{\mathrm{u}}\right)
\end{aligned}
$$

Onde,

$$
\lambda_{\mathrm{i}}\left(\alpha_{\mathrm{u}}\right)=\varphi\left(\xi Z_{\mathrm{i}} / \sigma_{\mathrm{u}}\right) / \Phi\left(\xi Z_{\mathrm{i}} / \sigma_{\mathrm{u}}\right)
$$

Donde $\varphi$ e $\Phi$ são, respectivamente, a função densidade de probabilidade e a função cumulativa de distribuição para uma distribuição normal. A razão $\lambda_{\mathrm{i}}$ (óu) é denominada a razão inversa de Mills.

Assim, dada a equação (9), agora se tem a regressão como:

$$
\mathrm{H}_{\mathrm{i}} \mid \mathrm{P}_{\mathrm{i}}^{*}>0=\beta^{\wedge} \mathrm{R}_{\mathrm{i}}+\beta \lambda_{\mathrm{i}}(\alpha \mathrm{u})+\varepsilon_{\mathrm{i}}
$$

A regressão em mínimos quadrados ordinários para as horas trabalhadas na semana, se levado em conta o vetor de variáveis independentes R e $\lambda$, será capaz de produzir estimativas consistentes para os parâmetros, 
utilizando os dados daqueles indivíduos que possuem salário de reserva menor do que aquele praticado pelo mercado.

Desse modo, o procedimento proposto por Heckman é capaz de produzir estimativas eficientes: o primeiro passo será regredir $\mathrm{P}$ em $\mathrm{Z}$ utilizando-se de um modelo Probit, onde $P$ define a participação do indivíduo no mercado de trabalho. De posse da estimativa de $\xi$ resultante desse modelo, é possível estimar $\lambda$ e, finalmente, através de mínimos quadrados ordinários, obtêm-se as estimativas de $\beta$.

Contudo, esse estimador ainda será heterocedástico, provocando erro na estimativa dos desvios dos parâmetros, resultando numa estatística t incorreta e prejudicando a interpretação da confiabilidade dos parâmetros. Consequentemente, a matriz de covariância proposta por White é útil para realizar a correta leitura dos desvios dos parâmetros, sendo consistente.

A matriz de covariância proposta por White (1980) torna-se consistente, como demonstra Cibriani-Neto e Góis (2002), utilizando-se o resíduo de mínimos quadrados, sendo igual a:

$\left(X^{\prime} \mathrm{X}\right)^{-1} \mathrm{X}^{\prime} \Theta \mathrm{X}\left(\mathrm{X}^{\prime} \mathrm{X}\right)^{-1}$

Onde,

$$
\Theta=\operatorname{diag}\left(\mathrm{e}_{1}^{2}, \mathrm{e}_{2}^{2}, \ldots, \mathrm{e}_{\mathrm{n}}^{2}\right)
$$

Desse modo, quando utilizada a matriz calculada na equação (12), denominada Matriz de Covariância de White, corrige-se a heterocedasticidade e pode-se realizar interpretações corretas a respeito da significância dos parâmetros.

\subsection{Dados}

A base de dados foi estabelecida a partir das informações contidas nos microdados da Pesquisa Nacional por Amostra de Domicílios (PNAD), coletados pelo Instituo Brasileiro de Geografia e Estatística (IBGE), relativos aos anos de 2001 a 2005. A partir dos microdados disponíveis, foram selecionadas todas as mulheres e todos os homens em idade ativa, ou seja, entre 10 e 65 anos, residentes nas regiões metropolitanas estudadas.

Considerando que a variável dependente do modelo corresponde ao logaritmo natural das horas trabalhadas na semana, selecionaram-se os indivíduos ocupados que declararam o número de horas trabalhadas na semana, tanto no trabalho principal, quanto nos outros trabalhos, bem como os indivíduos desocupados, uma vez que é possível incluí-los via procedimento de Heckman. Ao mesmo tempo, descartaram-se também os indivíduos sem declaração de horas trabalhadas, juntamente com 
aqueles que não declararam rendimentos provenientes do trabalho e anos de estudo, visto que correspondem a variáveis explicativas do modelo.

Uma vez que interessa ao modelo o total de horas ofertadas na semana, a variável horas trabalhadas na semana refere-se ao somatório das horas trabalhadas no trabalho principal, secundário e outros trabalhos. Após a realização dessa agregação, a variável foi transformada para o logaritmo natural. Já em relação aos rendimentos provenientes do trabalho, esses também correspondem à agregação dos rendimentos provenientes de todos os trabalhos realizados e encontra-se em bases semanais. $\mathrm{Na}$ medida em que os valores são coletados pela referência mensal, cabe transformá-los em rendimentos semanais. Para tal, os rendimentos mensais foram divididos por 4,5 (4 semanas e meia) e convertidos para o valor em reais de 2005 utilizando o IGP-DI da FGV como deflator.

Além do salário semanal (em reais) de 2005 e dos anos de estudo como proxy de educação, as outras variáveis independentes selecionadas foram a idade, o número de filhos morando com a mulher, bem como três variáveis Dummy para, respectivamente, condição de chefe de família, (i)migração e recebimento de outras rendas não provenientes do trabalho. Para chefe de familia a Dummy assume valor 1 se essa condição se confirma. Já para rendimentos fora do trabalho, a existência desses faz a Dummy assumir valor 1 para o indivíduo observado. Para (i)migração a Dummy assume valor 1 se o indivíduo reside a menos de cinco anos no município. Esta Dummy se justifica, pois muitos indivíduos deslocam-se para ofertar força de trabalho em outras cidades ou acompanhar parentes que o farão. Também foi introduzida uma Dummy relativa à ocorrência de trabalhos domésticos, parte integrante da PNAD, cujo valor 1 indica a presença de trabalho doméstico.

As tabelas 1.1, 1.2, 1.3, 2.1, 2.2 e 2.3, na sequência, apresentam uma estatística descritiva dos dados para as três regiões, dividida por gêneros, no qual inicialmente são apresentados os homens (tabelas 1.x) e posteriormente as mulheres (tabelas 2.x), ordenados pelas três regiões: Porto Alegre (y.1), Curitiba (y.2) e São Paulo (y.3).

Tabela 1.1 - Homens - Região Metropolitana de Porto Alegre

(34.470 observações; 24.264 observações não censuradas)

\begin{tabular}{c|c|c|c|c}
\hline Variáveis & Média & Mediana & Moda & Desvio Padrão \\
\hline Horas Trabalhadas na Semana de Referência & 31,82 & 40 & 0 & 23,37 \\
\hline Salário Hora (R\$2005) & 6,21 & 3,21 & 0 & 14,3749 \\
\hline Educação (anos de estudo) & 7,72 & 8 & 11 & 3,852 \\
\hline Idade & 32,86 & 32 & 21 & 14,92 \\
\hline Dummy Renda & 0,059 & 0 & 0 & 0,2355 \\
\hline \multicolumn{4}{|r}{}
\end{tabular}

MORAES, S. M. A.; MORAES, G. I. Oferta de trabalho comparada nas regiôes metropolitanas... 


\begin{tabular}{c|c|c|c|c}
\hline Dummy (i)migração & 0,1949 & 0 & 0 & 0,3961 \\
\hline Dummy Chefe Família & 0,5437 & 1 & 1 & 0,4981 \\
\hline Dummy Trabalho Doméstico & 0,6642 & 1 & 1 & 0,4723 \\
\hline
\end{tabular}

Fonte: IBGE - PNAD (2001, 2002, 2003, 2004, 2005).

Tabela 1.2 - Homens - Região Metropolitana de Curitiba (14.695 observações; 10.334 observações não censuradas)

\begin{tabular}{c|c|c|c|c}
\hline Variáveis & Média & Mediana & Moda & Desvio Padrão \\
\hline Horas Trabalhadas na Semana de Referência & 31,94 & 40 & 0 & 23,74 \\
\hline Salário Hora (R\$ 2005) & 6,41 & 3,36 & 0 & 16,04 \\
\hline Educação (anos de estudo) & 7,8 & 8 & 11 & 4,03 \\
\hline Idade & 31,9 & 30 & 23 & 14,49 \\
\hline Dummy Renda & 0,061 & 0 & 0 & 0,2388 \\
\hline Dummy (i)migração & 0,2531 & 0 & 0 & 0,4348 \\
\hline Dummy Chefe Família & 0,5330 & 1 & 1 & 0,4989 \\
\hline Dummy Trabalho Doméstico & 0,5794 & 1 & 1 & 0,4937 \\
\hline
\end{tabular}

Fonte: IBGE - PNAD (2001, 2002, 2003, 2004, 2005).

Tabela 1.3 - Homens - Região Metropolitana de São Paulo (39.239 observações; 25.655 observações não censuradas)

\begin{tabular}{c|c|c|c|c}
\hline Variáveis & Média & Mediana & Moda & Desvio Padrão \\
\hline Horas Trabalhadas na Semana de Referência & 29,88 & 40 & 0 & 23,96 \\
\hline Salário Hora (R\$ 2005) & 7,23 & 3,74 & 0 & 17,40 \\
\hline Educação (ano de estudo) & 7,87 & 8 & 11 & 4,01 \\
\hline Idade & 32,15 & 30 & 22 & 14,67 \\
\hline Dummy Renda & 0,0472 & 0 & 0 & 0,2121 \\
\hline Dummy (i)migração & 0,2879 & 0 & 0 & 0,4528 \\
\hline Dummy Chefe Família & 0,5075 & 1 & 1 & 0,5 \\
\hline Dummy Trabalho Doméstico & 0,4928 & 0 & 0 & 0,5 \\
\hline
\end{tabular}

Fonte: IBGE - PNAD (2001, 2002, 2003, 2004, 2005).

Tabela 2.1 - Mulheres - Região Metropolitana de Porto Alegre

(37.229 observações; 19.241 observações não censuradas)

\begin{tabular}{c|c|c|c|c}
\hline Variáveis & Média & Mediana & Moda & Desvio Padrão \\
\hline Horas Trabalhadas na Semana de Referência & 20,1 & 8 & 0 & 22,11 \\
\hline Salário Hora (R\$ 2005) & 4,8 & 2,66 & 0 & 7,85 \\
\hline Educação (anos de estudo) & 7,92 & 8 & 11 & 3,97 \\
\hline
\end{tabular}




\begin{tabular}{c|c|c|c|c}
\hline Idade & 34 & 33 & 21 & 15,03 \\
\hline Dummy Renda & 0,10 & 0 & 0 & 0,2995 \\
\hline Dummy Imigração & 0,1945 & 0 & 0 & 0,3961 \\
\hline Dummy Chefe Família & 0,2325 & 0 & 0 & 0,4224 \\
\hline Número de Filhos & 0,9685 & 1 & 0 & 1,1865 \\
\hline Dummy Trabalho Doméstico & 0,9159 & 1 & 1 & 0,2775 \\
\hline
\end{tabular}

Fonte: IBGE - PNAD (2001, 2002, 2003, 2004, 2005).

Tabela 2.2 - Mulheres - Região Metropolitana de Curitiba (15.407 observações; 7972 observações não censuradas)

\begin{tabular}{c|c|c|c|c}
\hline Variáveis & Média & Mediana & Moda & Desvio Padrão \\
\hline Horas Trabalhadas na Semana de Referência & 20,09 & 7 & 0 & 22,26 \\
\hline Salário Hora (R\$ 2005) & 4,47 & 2,57 & 0 & 7,76 \\
\hline Educação (anos de estudo) & 7,83 & 8 & 11 & 4,17 \\
\hline Idade & 33,16 & 32 & 20 & 14,59 \\
\hline Dummy Renda & 0,082 & 0 & 0 & 0,2754 \\
\hline Dummy Imigração & 0,261 & 0 & 0 & 0,4392 \\
\hline Dummy Chefe Família & 0,2046 & 0 & 0 & 0,4034 \\
\hline Número de Filhos & 1,0312 & 1 & 0 & 1,2077 \\
\hline Dummy Trabalho Doméstico & 0,90 & 1 & 1 & 0,30 \\
\hline
\end{tabular}

Fonte: IBGE - PNAD (2001, 2002, 2003, 2004, 2005).

Tabela 2.3 - Mulheres - Região Metropolitana de São Paulo (43.394 observações; 20.122 observações não censuradas)

\begin{tabular}{c|c|c|c|c}
\hline Variáveis & Média & Mediana & Moda & Desvio Padrão \\
\hline Horas Trabalhadas na Semana de Referência & 18,49 & 0 & 0 & 21,89 \\
\hline Salário Hora (R\$ 2005) & 5,62 & 3,05 & 0 & 17,06 \\
\hline Educação (anos de estudo) & 7,95 & 8 & 11 & 4,07 \\
\hline Idade & 33,3 & 32 & 22 & 14,74 \\
\hline Dummy Renda & 0,063 & 0 & 0 & 0,24 \\
\hline Dummy Imigração & 0,3047 & 0 & 0 & 0,4603 \\
\hline Dummy Chefe Família & 0,2091 & 0 & 0 & 0,4066 \\
\hline Número de Filhos & 1,03 & 1 & 0 & 1,24 \\
\hline Dummy Trabalho Doméstico & 0,888 & 1 & 1 & 0,3153 \\
\hline
\end{tabular}

Fonte: IBGE - PNAD (2001, 2002, 2003, 2004, 2005). 


\section{Estimação e Resultados}

A estimação da curva de oferta dos trabalhadores das três regiões metropolitanas escolhidas obedecerá ao enfoque de Heckman (1979) em dois estágios. No primeiro estágio, correspondentes a determinar a participação do trabalhador no mercado de trabalho, serão utilizadas com variáveis independentes (sinal esperado pela teoria): a idade (positivo) e seu quadrado (negativo), a educação (positiva) e seu quadrado, ${ }^{3}$ o número de filhos residentes, sendo esta variável apenas para as mulheres (negativo), a dummy correspondente a existência de outras rendas (negativo), a dummy de trabalho doméstico (negativo), a Dummy de imigração (positivo) e a Dummy correspondente a posição de chefe de família (positivo).

No segundo estágio da regressão, efetivo para determinar a oferta de trabalho, insere-se além da razão inversa de Mills $(\lambda)$, resultante do primeiro estágio, as seguintes variáveis independentes (sinal esperado pela teoria): a renda semanal (positivo) e seu quadrado (negativo), a idade (positiva) e seu quadrado (negativo) e o número de filhos residentes no mesmo lar apenas para as mulheres (negativo).

Reforça-se que a estimação em pooling para os 5 anos escolhidos também pressupõe que os parâmetros permanecem estáveis durante o período, ou seja, que não haveria fatores essenciais modificando significativamente o mercado de trabalho durante o período. Pode-se considerar, na ausência de reformas estruturais no mercado de trabalho, que a última grande influência sobre esse segmento foi a mudança da política cambial, ocorrida em 1999.

A opção em estimar as equações particularizadas por gêneros devese ao fato de que a variável número de filhos com os quais reside pode ser importante para o caso das mulheres e pela característica diferenciada entre os dois mercados de trabalho.

\subsection{Resultados para Homens}

As tabelas 3.1 e 3.2, abaixo, apresentam os resultados para os dois estágios de estimação para a oferta de trabalho dos homens nas regiões metropolitanas de Porto Alegre, Curitiba e São Paulo.

Para a decisão de participar do mercado de trabalho, as variáveis independentes apresentaram regra geral, os sinais esperados, mas algumas considerações podem ser feitas. O termo quadrático associado à educação não apresentou nas regiões metropolitanas de Porto Alegre e São Paulo

3 A princípio, espera-se que essa variável seja não significativa ou, ainda, que reforce o sinal positivo esperado para o primeiro polinômio. De qualquer forma, insere-se o polinômio de grau 2 para testar a hipótese de um ponto ótimo para essa variável. 
o sinal esperado, e na Região Metropolitana de Curitiba não se mostrou significativo. Com isso, pode-se concluir que elevados níveis de escolaridade contribuem para o ingresso no mercado de trabalho. A dummy de (i) migração na Região Metropolitana de Curitiba não se mostrou significativa, ao contrário das demais regiões. O ponto onde os homens passariam a diminuir sua disposição de ofertar trabalho para Porto Alegre localiza-se próximo aos 77 anos de idade, resultado idêntico para Curitiba e São Paulo. Como essas idades superam a expectativa de vida dessas regiões, trata-se de uma constatação teórica, deduzindo-se daí que empiricamente o salário praticado no mercado seria superior ao salário de reserva para todas as idades, coeteris paribus.

As dummies de imigração significativas para Porto Alegre e São Paulo justificam-se tendo em vista a decisão individual de deslocamento por melhores rendimentos, já estudada por diversos autores. ${ }^{4}$

Para a estimativa de horas ofertadas, dada a disposição em participar do mercado, chama novamente a atenção o comportamento da variável idade. As estimativas demonstram que há uma curva convexa para as regiões metropolitanas de Porto Alegre e São Paulo. Esse é um resultado contrário ao que se aguardava pela teoria, demonstrando que ao envelhecer o homem ofertaria mais horas de trabalho. Na Região Metropolitana de Curitiba, a variável idade e seu termo quadrático mostraram-se não significativas. Ressalta-se que ocorre o inverso para a decisão de participar ou não do mercado de trabalho (primeiro estágio), que apresenta uma curva côncava, conforme esperado. Para resultados particulares das regiões, o ponto ótimo para a oferta de horas de trabalho dá-se com salários de $R \$ 53.435,89, R \$ 17.506,56$ e $R \$ 16.966,29$, respectivamente, para as regiões metropolitanas de Porto Alegre, Curitiba e São Paulo. Dessa forma, é possível perceber que a região metropolitana de Porto Alegre diferencia-se nesse particular.

Tabela 3.1 - Variável Dependente: Trabalha ou Não? ( $1^{\circ}$ Estágio)

\begin{tabular}{c|c|c|c}
\hline $\begin{array}{c}\text { Coeficiente } \\
\text { (Desvio Padrão) }\end{array}$ & Porto Alegre & Curitiba & São Paulo \\
\hline Constante & $\begin{array}{c}-3,746269 \\
(0,0578233)\end{array}$ & $\begin{array}{c}-3,642152 \\
(0,0885136)\end{array}$ & $\begin{array}{c}-3,656739 \\
(0,0550128)\end{array}$ \\
\hline \multirow{2}{*}{ Idade } & 0,2444342 & 0,2404711 & 0,224071 \\
& $(0,0033058)$ & $(0,005098)$ & $(0,0030759)$ \\
\hline \multirow{2}{*}{ Idade $^{2}$} & $-0,0031585$ & $-0,0031315$ & $-0,0029125$ \\
& $(0,000043)$ & $(0,0000668)$ & $(0,0000399)$ \\
\hline
\end{tabular}

4 Destacadamente Harris e Todaro (1970).

MORAES, S. M. A.; MORAES, G. I. Oferta de trabalho comparada nas regiôes metropolitanas... 
(continuação)

\begin{tabular}{c|c|c|c}
\hline Educação & $\begin{array}{c}0,0361046 \\
(0,0087861)\end{array}$ & $\begin{array}{c}0,0478719 \\
(0,0126065)\end{array}$ & $\begin{array}{l}0,0174345 \\
(0,007444)\end{array}$ \\
\hline $\begin{array}{c}0,0006097 \\
(0,000552)\end{array}$ & $\begin{array}{c}-0,000584^{*} \\
(0,0008052)\end{array}$ & $\begin{array}{c}0,0034999 \\
(0,0004789)\end{array}$ \\
\hline $\begin{array}{c}\text { Dummycação } \\
\text { Outras Rendas }\end{array}$ & $\begin{array}{c}-0,5398111 \\
(0,034578)\end{array}$ & $\begin{array}{c}-0,3849708 \\
(0,053917)\end{array}$ & $\begin{array}{c}-0,4048524 \\
(0,035863)\end{array}$ \\
\hline $\begin{array}{c}\text { Dummy } \\
\text { Chefe de Família }\end{array}$ & $\begin{array}{c}0,5538672 \\
(0,0236205)\end{array}$ & $\begin{array}{c}0,6228382 \\
(0,0361197)\end{array}$ & $\begin{array}{c}0,5570215 \\
(0,0215316)\end{array}$ \\
\hline Dummy Imigração & $\begin{array}{c}0,1011903 \\
(0,0222915)\end{array}$ & $\begin{array}{c}-0,0166632^{*} \\
(0,0303477)\end{array}$ & $\begin{array}{c}0,1439868 \\
(0,0176754)\end{array}$ \\
\hline $\begin{array}{c}\text { Dummy } \\
\text { Trabalho Doméstico }\end{array}$ & $\begin{array}{c}-0,1247379 \\
(0,0184151)\end{array}$ & $\begin{array}{c}-0,2018619 \\
(0,0268552)\end{array}$ & $\begin{array}{c}-0,2639881 \\
(0,0157237)\end{array}$ \\
\hline $\begin{array}{c}\text { Número de } \\
\text { Observações }\end{array}$ & 34.470 & 14.695 & 39.239 \\
\hline $\begin{array}{c}\text { Observações } \\
\text { Censuradas } \\
\text { (Não Trabalha) }\end{array}$ & 10.206 & 4.361 & 13.584 \\
\hline $\begin{array}{c}\text { Observações } \\
\text { Não Censuradas } \\
\text { (Trabalha) }\end{array}$ & 24.264 & 10.334 & 25.655 \\
\hline
\end{tabular}

*- não significativa a 5\%

Fonte: Dados da pesquisa a partir dos microdados da PNAD.

Tabela 3.2 - Variável Dependente: Logaritmo das Horas de Trabalho Ofertadas na Semana ( $2^{\circ}$ estágio)

\begin{tabular}{c|c|c|c}
\hline $\begin{array}{c}\text { Coeficiente } \\
\text { (Desvio Padrão) }\end{array}$ & Porto Alegre & Curitiba & São Paulo \\
\hline Constante & $\begin{array}{c}4,058695 \\
(0,0585677)\end{array}$ & $\begin{array}{c}4,031519 \\
(0,0898078)\end{array}$ & $\begin{array}{c}3,641882 \\
(0,0423938)\end{array}$ \\
\hline Idade & $-0,0101964$ & $-0,0086104^{*}$ & 0,0099824 \\
& $(0,0029496)$ & $(0,0045593)$ & $(0,0021124)$ \\
\hline Idade ${ }^{2}$ & 0,000128 & $0,0001079^{*}$ & $-0,0001143$ \\
& $(0,0000375)$ & $(0,0000582)$ & $(0,0000271)$ \\
\hline Salário & 0,0000521 & 0,0000667 & 0,0000302 \\
& $\left(7,47 \mathrm{e}^{-06}\right)$ & $(0,0000121)$ & $\left(6,01 \mathrm{e}^{-06}\right)$ \\
\hline Salário ${ }^{2}$ & $-9,75 \mathrm{e}^{-10}$ & $-3,81 \mathrm{e}^{-09}$ & $-1,78 \mathrm{e}^{-09}$ \\
$\left(2,74 \mathrm{e}^{-10}\right)$ & $\left(1,04 \mathrm{e}^{-09}\right)$ & $\left.-0,40 \mathrm{e}^{-10}\right)$ \\
\hline$\lambda$ (Razão Inversa & $-0,4323583$ & $-0,4378019$ & $-0,1796028$ \\
de Mills) & $(0,0209498)$ & $(0,031808)$ & $(0,0145312)$ \\
\hline Wald $\chi^{2}$ Statistic & 5565,41 & 2276,82 & 5477,86 \\
\hline
\end{tabular}

*- não significativa a $5 \%$

Fonte: Dados da pesquisa a partir dos microdados da PNAD. 


\subsection{Resultados para Mulheres}

As tabelas 4.1 e 4.2 explicitam os dois estágios de estimação para a oferta de trabalho das mulheres para as regiões metropolitanas de Porto Alegre, Curitiba e São Paulo.

Para a decisão de participar ou não do mercado de trabalho e por conseqüência determinar se o salário de reserva é menor que o ofertado no mercado, a estimativa apresentou os sinais esperados, mas a Dummy de (i)migração para Porto Alegre não se mostrou significativa, tal como a variável educação para Curitiba. De qualquer forma, nesse último caso, a variável educação na sua forma quadrática apresentou-se significativa e com o sinal esperado. A variável número de filhos residentes com a mulher, disponível na PNAD, demonstrou-se importante para as três regiões metropolitanas. Comparadas aos homens, as mulheres apresentam uma idade mais elevada para (teoricamente) deixarem o mercado de trabalho. Em Porto Alegre, a idade ótima é próxima dos 78 anos e em Curitiba e São Paulo, dos 77 anos. $^{5}$

É importante salientar que o sinal significativo para a variável educação no primeiro estágio de estimação confirma os resultados obtidos por Scorzafave e Menezes-Filho (2001) para o período entre 1982 e 1997, no qual concluem que a educação é uma variável independente chave para explicar a participação da mulher no mercado de trabalho, recorrendo à decomposição de efeitos de Yun e Oaxaca-Blinder.

Barros et al. (1994) também percebem, recorrendo à decomposição de efeitos, a educação como a variável chave para explicar a participação crescente da mulher no mercado de trabalho entre as décadas de 50 e 80. Soares e Izaki (2002) também observam o mesmo comportamento definindo como elo causal a maior escolaridade feminina como fator determinante.

Para a oferta de horas, estabelecida a disposição em participar, a variável número de filhos também é significativa. Por sua vez, a idade é uma variável significativa e forma uma curva côncava em relação à oferta de horas. Na Região Metropolitana de Curitiba, todavia, a variável idade em seu polinômio de primeiro grau não é considerada significativa a 5\% de confiança, mas somente a 10\%. Para a variável salário, os sinais foram significativos e apresentam os sinais esperados pela teoria. Além disso, é importante notar que os níveis de ponto ótimo nas horas ofertadas foram inferiores aos dos homens. Para Porto Alegre, esse nível de salário é de $\mathrm{R} \$ 3.194,31$, para Curitiba $\mathrm{R} \$ 5.385,71$ e para São Paulo $\mathrm{R} \$ 10.284,67 .^{6}$

5 Mais uma vez as idades ótimas superam a expectativa de vida, constituindo-se em um resultado teórico.

6 Recordando-se que se trata de salário semanal. Logo, o ponto ótimo para a imensa maioria das trabalhadoras constitui-se numa miragem teórica. 
Avelino e Menezes-Filho (2003) pesquisaram a variável número de filhos, incluindo dummies para as faixas etárias desses. Eles concluíram que a presença de filhos, de fato, reduz a oferta de horas de trabalho para as mulheres. Diferentemente do presente artigo, naquele os autores trabalham com a oferta intertemporal de trabalho, proposta por Heckman e MaCurdy (1980). O mesmo resultado é obtido por Pazello (2006), limitando-se, entretanto, a influência da variável apenas sobre a decisão de participação no mercado de trabalho.

Tabela 4.1 - Variável Dependente: Trabalha ou Não? (1 ${ }^{\circ}$ Estágio)

\begin{tabular}{|c|c|c|c|}
\hline $\begin{array}{c}\text { Coeficiente } \\
\text { (Desvio Padrão) }\end{array}$ & Porto Alegre & Curitiba & São Paulo \\
\hline Constante & $\begin{array}{c}-3,577415 \\
(0,0572927)\end{array}$ & $\begin{array}{c}-3,151964 \\
(0,0853851)\end{array}$ & $\begin{array}{c}-3,273573 \\
(0,0530078)\end{array}$ \\
\hline Idade & $\begin{array}{c}0,2102499 \\
(0,0031575)\end{array}$ & $\begin{array}{c}0,1997785 \\
(0,0048685)\end{array}$ & $\begin{array}{c}0,1986287 \\
(0,0030251)\end{array}$ \\
\hline Idade $^{2}$ & $\begin{array}{l}-0,0026928 \\
(0,0000416)\end{array}$ & $\begin{array}{c}-0,002586 \\
(0,0000647)\end{array}$ & $\begin{array}{l}-0,0025682 \\
(0,0000399)\end{array}$ \\
\hline Educação & $\begin{array}{c}0,023299 \\
(0,0072707)\end{array}$ & $\begin{array}{l}-0,0111732^{*} \\
(0,0102575)\end{array}$ & $\begin{array}{l}-0,0186584 \\
(0,0062904)\end{array}$ \\
\hline Educação ${ }^{2}$ & $\begin{array}{c}0,0019372 \\
(0,0004399)\end{array}$ & $\begin{array}{c}0,0039242 \\
(0,0006467)\end{array}$ & $\begin{array}{c}0,0055901 \\
(0,0003933)\end{array}$ \\
\hline $\begin{array}{c}\text { Dummy } \\
\text { Outras Rendas }\end{array}$ & $\begin{array}{l}-0,2205099 \\
(0,0240935)\end{array}$ & $\begin{array}{l}-0,1792752 \\
0,0398501\end{array}$ & $\begin{array}{c}-0,1974084 \\
0,0272239\end{array}$ \\
\hline $\begin{array}{c}\text { Dummy } \\
\text { Chefe de Família }\end{array}$ & $\begin{array}{c}0,3326257 \\
(0,0179218)\end{array}$ & $\begin{array}{c}0,296505 \\
(0,0285288)\end{array}$ & $\begin{array}{c}0,4285716 \\
(0,0169911)\end{array}$ \\
\hline $\begin{array}{l}\text { Número de Filhos } \\
\text { Morando no Lar }\end{array}$ & $\begin{array}{l}-0,1042269 \\
(0,0069886)\end{array}$ & $\begin{array}{l}-0,1142796 \\
(0,0107136)\end{array}$ & $\begin{array}{l}-0,1313798 \\
(0,0063392)\end{array}$ \\
\hline Dummy Imigração & $\begin{array}{l}0,0236879^{*} \\
(0,0176622)\end{array}$ & $\begin{array}{c}-0,0731402 \\
(0,0246678)\end{array}$ & $\begin{array}{c}0,0837406 \\
(0,0145291)\end{array}$ \\
\hline $\begin{array}{c}\text { Dummy } \\
\text { Trabalho Doméstico }\end{array}$ & $\begin{array}{l}-0,1429823 \\
(0,0282955)\end{array}$ & $\begin{array}{l}-0,2042325 \\
(0,0397108)\end{array}$ & $\begin{array}{l}-0,3751847 \\
(0,023384)\end{array}$ \\
\hline $\begin{array}{l}\text { Número de } \\
\text { Observações }\end{array}$ & 37.229 & 15.407 & 43.394 \\
\hline $\begin{array}{c}\text { Observações } \\
\text { Censuradas } \\
\text { (Não Trabalha) }\end{array}$ & 17.988 & 7.435 & 23.272 \\
\hline $\begin{array}{l}\text { Observações } \\
\text { Não Censuradas } \\
\text { (Trabalha) }\end{array}$ & 19.241 & 7.972 & 20.122 \\
\hline
\end{tabular}

*- não significativa a $5 \%$

Fonte: Dados da pesquisa a partir dos microdados da PNAD. 
Tabela 4.2 - Variável Dependente: Logaritmo das Horas de Trabalho Ofertadas na Semana ( $2^{\circ}$ estágio)

\begin{tabular}{c|c|c|c}
\hline $\begin{array}{c}\text { Coeficiente } \\
\text { (Desvio Padrão) }\end{array}$ & Porto Alegre & Curitiba & São Paulo \\
\hline Constante & $\begin{array}{c}3,281225 \\
(0,0942489)\end{array}$ & $\begin{array}{c}3,423124 \\
(0,1535151)\end{array}$ & $\begin{array}{c}3,614422 \\
(0,0615363)\end{array}$ \\
\hline Idade & 0,0185581 & 0,0143716 & 0,005967 \\
& $(0,0045053)$ & $(0,0074444)$ & $(0,0030423)$ \\
\hline Idade ${ }^{2}$ & $-0,0003001$ & $-0,0002407$ & $-0,0001206$ \\
Salário & $(0,0000581)$ & $(0,0000976)$ & $(0,0000397)$ \\
\hline Salário ${ }^{2}$ & 0,0007858 & 0,0006786 & 0,0002818 \\
\hline Número de Filhos & $(0,0000311)$ & $(0,0000458)$ & $(0,0000163)$ \\
Morando no Lar & $-2.46 \mathrm{e}^{-07}$ & $-1.26 \mathrm{e}^{-07}$ & $-2.74 \mathrm{e}^{-08}$ \\
\hline$\lambda($ Razão Inversa & $\left(1,37 \mathrm{e}^{-08}\right)$ & $\left(1.23 \mathrm{e}^{-08}\right)$ & $-0,0173522$ \\
de Mills) & $-0,0248698$ & $-0,0262502$ & $(0,003714)$ \\
\hline Wald $\chi^{2}$ Statistic & $(0,0044886)$ & $(0,0077801)$ & $-0,133755$ \\
\hline
\end{tabular}

*- não significativa a $5 \%$

Fonte: Dados da pesquisa a partir dos microdados da PNAD.

\section{Considerações Finais}

O contrato de trabalho efetuado no Brasil, na grande maioria dos casos, prevê que o número fixo de horas semanais seja de 40 horas. Isso torna pouco flexível a opção por parte dos agentes de definir o número de horas de trabalho a sua escolha. Assim, os resultados desta pesquisa, que fez uso dos microdados da PNAD de 2001 a 2005, encontram limitação nesse aspecto das práticas brasileiras. Por essa razão, percebe-se que a diferença entre a oferta de trabalho das três regiões metropolitanas é mínima, constituindo-se em um mercado de trabalho homogêneo no que diz respeito à decisão do agente frente ao mercado de trabalho.

As variáveis independentes escolhidas, regra geral, mostraram-se significativas, e chama atenção o fato de que a variável número de filhos é significativa para determinar se a mulher participa ou não do mercado de trabalho e decisiva também para dizer quantas horas serão ofertadas.

A estimação também confirmou a importância na decisão de ofertar horas de trabalho da variável (i)migração. Confirma-se que o deslocamento de residência, na maior parte das vezes, diz respeito ao mercado de trabalho e suas oportunidades. A condição de chefe de família é outra das 
variáveis que mostra que trabalhadores em posição principal na família tendem a ingressar no mercado de trabalho, ratificando a teoria. Da mesma forma, a existência de outras fontes de renda, a existência de trabalho no lar, idade e nível educacional são igualmente variáveis importantes.

\section{Referências}

AVELINO, R.; MENEZES-FILHO, N. Estimação da Oferta de Trabalho das Mulheres no Brasil. São Paulo: Estudos Econômicos, v. 33, n. 4, p. 639-664, 2003.

BARBOSA, F. H. Microeconomia: Teoria, Modelos Econométricos e Aplicaçõs à Economia Brasileira. Rio de Janeiro: PNPE/IPEA, 1985.

BARROS, L.; BRITTO, R. O Funcionamento do Mercado de Trabalho Metropolitano Brasileiro no Período 1991-2002: Tendências, Fatos Estilizados e Mudanças Estruturais. Rio de Janeiro: IPEA, Texto Para Discussão n. 1.011, 2004.

BARROS, R.; JATOBÁ, J.; MENDONÇA, R. A Evolução da Participação das Mulheres no Mercado de Trabalho: Uma Análise de Decomposição. XVI Encontro Brasileiro de Econometria, Sociedade Brasileira de Econometria, Florianópolis, 1994.

CIBRIANI-NETO, F.; GÓIS, M. C. A. Uma Análise de Monte Carlo do Desempenho dos Estimadores de Matrizes de Covariância sob Heterocedasticidade de Forma Desconhecida. Revista Brasileira de Economia, v. 56, n. 2, p. 309-34, 2002.

DEATON, A.; MUELLBAUER, J. Economics and Consumer Behavior. Cambridge: Cambridge University Press, 1991.

DONNI, O. On the Indetification of Frisch Labor Supplies. CIRPEÉ: Centre Interuniversitarie sur le risqué, les politiques économiques et l'employ: Cahier de Researche. 2006, p 06-20.

HARRIS, J.; TODARO, M. Migration, Uneployment And Development: A Two SectorAnalysis. American Economic Review, 60, p. 126-142, 1970.

HECKMAN, J. J. Shadow prices, market wages, and labor supply, Econométrica, 1974, p. 679-694.

. Sample selection bias as a specification error, Econométrica, 47, p. 153-162, 1979.

. Sample Selection Bias as a Specification Error. In: Smith, J. P. (ed.). Female Labor $\overline{S u p p l y}$ : theory and estimation. Princeton (NJ): Princeton University Press, 1980.

HECKMAN, J. J.; MaCURDY, T. A Life Cycle Model of Female Labor Supply. Review of Economic Studies, v. 47, p. 47-74, 1991.

IBGE - Instituto Brasileiro de Geografia e Estatística. Produto Bruto dos Municípios 2004 Contas Nacionais $n^{\circ}$ 18. Rio de Janeiro: IBGE, 2006. Disponível em: <http://www.ibge. gov.br/home/estatistica/economia/pibmunicipios/2004/pibmunic2004.pdf > .

.PNAD - Pesquisa Nacional de Amostras por Domicílio - Microdados. Rio de Janeiro:
IBGE, Vários Anos.

IPEADATA. Dados Estatísticos da Economia Brasileira. Disponível em: <www.ipeadata. gov.br/>.

KASSOUF, A. L. The wage rate estimation using the Heckman procedure, Revista de Econometria, 1994, p. 89-107. 
KASSOUF, A. L. Trabalho Infantil no Brasil. Tese de Livre Docência. ESALQ/USP, Piracicaba, SP, 1999.

KILLINGSWORTH, M.; HECKMAN, J. Female Labor Supply. Handbook of Labor Economics, In: ASHENFELTER, O.; LAYARD, R. (orgs.). Amsterdam: ElSevier Science Publishers, 1986, p. 103-204.

PAZELLO, E. T. A Maternidade Afeta o Engajamento da Mulher no Mercado de Trabalho? Um Estudo Utilizando o Nascimento de Gêmeos como um Experimento Natural. São Paulo: Estudos Econômicos, v. 36, n. 3, p. 507-538, 2006.

RAMOS, L.; BRITTO, M. O Funcionamento do Mercado de Trabalho Metropolitano Brasileiro no Período 1991-2002: Tendências, Fatos Estilizados e Mudanças Estruturais. Texto para Discussão, n. 1.011, Rio de Janeiro: IPEA, 2004.

REZENDE, M.; WYLLIE, R. Retornos para Educação no Brasil. Economia Aplicada, v. 10, n. 3, p. 349-365, 2006.

SCORZAFAVE, L. G.; MENEZES-FILHO, N. A. A Participação Feminina no Mercado de Trabalho Brasileiro: Evolução e Determinantes. Pesquisa e Planejamento Econômico, v. 31, n. 3, p. 441-78, 2001.

SOARES, S.; IZAKI, R. S. A participação feminina no mercado de trabalho. Texto para Discussão, n. 293. Rio de Janeiro: IPEA, 2002.

WHITE, $\mathrm{H}$. A heteroskesdaticity consistent covariance matrix estimator and a direct test for heteroskedasticity. Econométrica, v. 48, n. 4, p. 827-838, 1980.

ZYLBERSTAJN, H.; BALBINOTTO NETO, G. As Teorias de Desemprego e as Políticas Públicas de Emprego. Estudos Econômicos, v. 29, n. 1, p. 129-149, 1999.

Recebido em: 22/07/2008. Aceito em: 24/08/2008. 